\title{
L'ALEXITHYMIE : CLÉ DE COMPRÉHENSION OU OBSTACLE À L'ACCOMPAGNEMENT DES AUTEURS DE VIOLENCE CONJUGALE ? VIGNETTES CLINIQUES
}

Cécile Kowal, Fabienne Hodiaumont, Laetitia Di Piazza, Adélaïde Blavier, Suzanne Léveillée, Carolanne Vignola-Lévesque et Robert Ayotte

Groupe d'études de psychologie | « Bulletin de psychologie »

2020/2 Numéro 566 | pages 115 à 128

ISSN 0007-4403

Article disponible en ligne à l'adresse :

https://www.cairn.info/revue-bulletin-de-psychologie-2020-2-page-115.htm

Distribution électronique Cairn.info pour Groupe d'études de psychologie.

(C) Groupe d'études de psychologie. Tous droits réservés pour tous pays.

La reproduction ou représentation de cet article, notamment par photocopie, n'est autorisée que dans les limites des conditions générales d'utilisation du site ou, le cas échéant, des conditions générales de la licence souscrite par votre établissement. Toute autre reproduction ou représentation, en tout ou partie, sous quelque forme et de quelque manière que ce soit, est interdite sauf accord préalable et écrit de l'éditeur, en dehors des cas prévus par la législation en vigueur en France. Il est précisé que son stockage dans une base de données est également interdit. 


\title{
L'alexithymie : clé de compréhension ou obstacle à l'accompagnement des auteurs de violence conjugale ? Vignettes cliniques
}

\author{
Kowal Cécile a \\ Hodiaumont Fabienne a \\ Di Piazza Laetitia ${ }^{b}$ \\ BLAVIER Adélaïde ${ }^{b}$ \\ LÉVEILlÉE Suzanne ${ }^{c}$ \\ Vignola-Lévesque Carolanne ${ }^{c}$ \\ AYotTe Robert $^{\mathrm{d}}$
}

\footnotetext{
a Praxis, association sans but lucratif, rue Puitsen-Sock, 63 Bte 22/32, 4020 Liège, Belgique.

b Centre d'expertise en psychotraumatismes et psychologie légale, Faculté de psychologie, logopédie et sciences de l'éducation, Université de Liège, Belgique.

c Département de psychologie, Université du Québec à Trois-Rivières, Canada.

d L'Accord Mauricie Inc., rue Champflour, 991 Local 101, Trois-Rivières, Québec, Canada.

Correspondance : Cécile Kowal, rue Puits-enSock, 63 Bte 22/32, 4020 Liège, Belgique. Courriel : cecile.kowal@asblpraxis.be Texte reçu le 23 novembre 2018 et accepté le 4 novembre 2019

http://www.bulletindepsychologie.net
}

Résumé : L'article présente quatre vignettes cliniques examinant l'expression et l'évolution de l'alexithymie chez les usagers d'un service belge, Praxis, qui propose un programme de responsabilisation en groupe pour auteurs de violence conjugale. Ces vignettes sont issues et viennent compléter les résultats d'une recherche longitudinale qui réunit des associations de terrain et des chercheurs universitaires, tant au Québec qu'en Belgique, et dont l'objectif est d'évaluer le changement psychologique chez ces hommes au terme de leur prise en charge thérapeutique. Les résultats montrent une prévalence des troubles alexithymiques et dépressifs chez les participants, en comparaison avec la population générale. La discussion des vignettes fait ressortir une hypothèse majeure relative à l'éventuelle distinction entre la présence d'une alexithymie primaire et d'une alexithymie secondaire.

Intimate partner violence: Is alexithymia a key to understanding a male offender's violence? Clinical cases

Abstract: Based on four clinical cases, this article aims to link the findings of a study led by the University of Liège, which was carried out to explore psychological changes in male perpetrators of domestic violence, and the actors involved in specific treatment programs. It focuses on the expression and evolution of alexithymia and its association with impulsivity and depression among users of a Belgian service, Praxis, which offers a group empowerment program for perpetrators of domestic violence. The findings indicated that male offenders reported more depressive feelings and more difficulty in identifying and expressing their emotions (alexithymia) than the general population. The discussion of clinical cases highlights an important hypothesis regarding the possible distinction between the presence of primary and secondary alexithymia. 


\section{INTRODUCTION}

Cet article a pour objectif de présenter les enjeux psychologiques ainsi que les changements psychologiques de quatre hommes auteurs de violence conjugale. Le choix des vignettes cliniques a été fait à partir d'une étude plus large visant une meilleure compréhension du changement psychologique d'hommes auteurs de violence conjugale. Le changement psychologique correspond à la présence de différences chez un individu dans ses attitudes, ses comportements, ses pensées ou réactions, qui lui permettent de mieux se sentir, de mieux se comprendre et de résoudre des problèmes (Ordre des psychologues au Québec, 2019). La présentation des vignettes cliniques et leur discussion sont précédées d'une définition du concept d'alexithymie en articulation avec les facteurs de risque connus dans le domaine des violences domestiques et le travail en groupe de responsabilisation, conduit au sein de Praxis, partenaire belge de la recherche.

\section{L'ALEXITHYMIE, UNE CLÉ DE COMPRÉHENSION}

\section{Éléments de définition}

« L'alexithymie signifie étymologiquement l'incapacité à exprimer ses émotions par des mots. Le concept se définit par quatre dimensions cliniques : l'incapacité à identifier et à exprimer verbalement ses émotions et ses sentiments, la limitation de la vie imaginaire, une pensée à contenu pragmatique accompagnée d'un mode d'expression très descriptif abordant plus volontiers les aspects triviaux des événements vécus sans véritable élaboration, et enfin le recours à l'action pour éviter les conflits ou exprimer les émotions » (Corcos, Pirlot, Loas, 2011, p. 25). Étant donné ces caractéristiques, les individus alexithymiques présentent une pauvreté dans leur capacité d'empathie. Corcos et coll. (2011, p. 26) ajoutent que «s sa fréquence dans la population générale est variable selon les études, mais serait estimée à environ $10 \%$ ». Jouanne (2006) retient une prévalence de $20 \%$ dans la population générale.

Plusieurs auteurs, dont Jouanne (2006) et Tychey (2010), citent Freyberger (1977), qui distingue deux approches étiologiques divergentes concernant l'alexithymie. L'une correspond à un « modèle déficitaire ». Au sein de ce modèle, l'alexithymie est définie comme un déficit structuré et durable $\mathrm{du}$ fonctionnement préconscient. L'alexithymie est alors considérée comme un trait structurel de la personnalité difficilement réversible. L'autre approche réfère à un " modèle de fonctionnement défensif ». Les auteurs définissent l'alexithymie comme un fonctionnement psychique régressif permettant un verrouillage des affects face à des situations de stress aigu ou des situations traumatisantes que l'individu ne parvient pas à élaborer (Bréjard, Bonnet, Pedinielli, 2008).

\section{Alexithymie ou défaut de mentalisation ?}

L'alexithymie comporte deux dimensions : l'une émotionnelle, l'autre cognitive. Ainsi, le processus de mentalisation serait un concept complémentaire nécessaire à la compréhension des phénomènes de pensées opératoires et du déficit de la vie fantasmatique observés chez les personnes présentant de l'alexithymie. Bateman et Fonagy (2015) définissent la mentalisation comme le mécanisme mental par lequel un individu interprète implicitement ou explicitement ses actions et celles des autres comme ayant un sens sur la base d'états mentaux intentionnels comme les désirs, les besoins, les sentiments, les croyances et les raisons. En effet, la mentalisation est une capacité qui permet à l'individu de donner du sens et d'intégrer un événement vécu en l'assimilant dans son psychisme. En cela, cet événement devient une représentation à partir de laquelle l'individu peut comprendre et organiser son monde. Mentaliser, c'est également renoncer au contrôle totalitaire de ses émotions et réactions en y apportant de la nuance et en tolérant les incertitudes inhérentes à l'existence de tout individu.

Selon Corcos, Spéranza et Jeammet (2003), l'alexithymie se caractérise par une carence des capacités de mentalisation, ce qui provoque un risque de décharge dans l'agir. Plusieurs études (Léveillée et coll., 2013 ; Adshead et coll., 2013 ; Di Piazza et coll., 2017 ; Léveillée et coll., 2009) confirment que la violence conjugale ne serait pas tant liée à des problèmes d'impulsivité motrice ou cognitive incontrôlable, mais plutôt à des difficultés de mentalisation. Elle proviendrait d'une stratégie d'action inappropriée visant le contrôle de l'autre.

\section{Alexithymie et conduites à risque}

Il existe actuellement peu d'études qui visent à comprendre les liens entre l'alexithymie et les comportements transgressifs, délinquants ou autres passages à l'acte autodestructeurs. Dans une étude réalisée auprès de 259 sujets, Bréjard et coll. (2008) testent l'hypothèse des relations entre fonctionnement émotionnel, trouble thymique et conduites à risque. Les résultats montrent que le noyau psychopathologique des conduites à risque semble être constitué de deux facteurs : la difficulté à identifier les émotions et le vécu dépressif. En revanche, dans cette étude, la composante cognitive de l'alexithymie ne représente pas une composante explicative des conduites à risque. 
Plusieurs auteurs rapportent des liens entre l'alexithymie et les troubles de dépendance (Guilbaud et coll., 2002), la dépression (Honkalampi et coll., 2000) et les délinquants violents incarcérés (Maisondieu, Tarrieu, Razafimamonjy, Arnault, 2008). Lorsque Jouanne (2006) montre une corrélation positive entre l'alexithymie et la dépendance alcoolique, ses constatations peuvent être transposées aux usagers des programmes d'aide aux conjoints violents. En effet, l'auteur indique que ces individus manquent d'un sentiment de cohésion de soi et de capacité à réguler leurs affects de façon autonome. Les séparations deviennent des périodes de grande vulnérabilité, durant lesquelles les sujets qui présentent de l'alexithymie établissent des relations de dépendance avec des personnes extérieures jouant le rôle de régulateurs ou avec des objets addictifs.

\section{Alexithymie et violence conjugale}

La violence conjugale réfère à la violence ou à la maltraitance exercée par le partenaire amoureux pendant la relation conjugale, lors de la rupture ou à la suite d'une rupture amoureuse. La violence est utilisée dans le but de contrôler le partenaire et peut prendre différentes formes, telles que la violence sexuelle et l'exploitation physique, psychologique, économique et spirituelle (Ministère de la Sécurité publique du Québec, 2013). Des études montrent que la présence d'alexithymie pourrait affecter la qualité des relations conjugales (Frye-Cox, Hesse, 2013). En ce sens, le concept d'alexithymie permettrait de donner un éclairage différent à ces observations cliniques sur la régulation émotionnelle et la difficulté à se maintenir dans un processus d'aide. En effet, selon Ogrodniczuk, Sochting, Piper et Joyce (2012), face au sujet alexithymique, le thérapeute est parfois confronté à l'absence de progrès et d'expressions émotionnelles positives durant les séances. Jouanne (2006) cite également plusieurs études qui montrent combien l'alexithymie apparaît comme un facteur de risque intéressant l'engagement dans un traitement : l'alliance thérapeutique se révèle plus fragile, le patient participe à moins de séances et s'y engage moins. Ainsi, l'alexithymie serait une variable majeure afin de mieux comprendre le fonctionnement de ces hommes. L'étude de Léveillée et coll. (2013) a permis d'évaluer le changement psychologique d'hommes auteurs de violence conjugale et qui consultaient dans un organisme spécialisé afin d'aider les personnes aux prises avec cette problématique. Selon leurs résultats, il y a présence d'alexithymie chez plus de la moitié des hommes auteurs de violence conjugale. Par ailleurs, les résultats indiquent une diminution significative de l'alexithymie au terme de la thérapie.
Plus récemment, les résultats de l'étude de Di Piazza et coll. (2017) indiquent que les auteurs de violence conjugale obtiennent des scores plus élevés aux échelles d'alexithymie que la population générale. En effet, $45 \%$ des hommes auteurs de violence conjugale présentent des scores correspondant à la présence d'alexithymie. En outre, $21 \%$ des participants présentent un fonctionnement indéterminé ou sub-alexithymique. En d'autres termes, leur fonctionnement psychologique est tantôt empreint d'alexithymie, tantôt exempt de cette incapacité émotionnelle. Au total, il s'agit de $66 \%$ de l'échantillon. Parallèlement, les hommes qui commettent des comportements violents contre leur partenaire ont tendance à rapporter plus de symptômes dépressifs que les individus provenant de la population générale. En effet, $70 \%$ des participants obtiennent un score correspondant à la présence d'une humeur dépressive, qu'elle soit légère (trouble bénin de l'humeur : $35 \%)$ ou modérée à sévère (35\%). Contrairement aux deux caractéristiques précédentes, l'impulsivité ne semble pas caractériser cette population : $59 \%$ des hommes ayant participé à l'étude obtiennent des scores indiquant une absence d'impulsivité et seuls $25 \%$ des auteurs sont considérés impulsifs. Il existe, chez les hommes auteurs de violence conjugale, un lien positif entre l'alexithymie, l'impulsivité et la dépression. Ainsi, plus les participants présentent des affects dépressifs et ont des difficultés à identifier et à décrire leurs émotions, plus leur comportement est empreint d'impulsivité.

\section{Objectifs de la présente étude}

La présente étude vise à évaluer trois caractéristiques psychologiques, soit les affects dépressifs, l'impulsivité et l'alexithymie, chez quatre hommes auteurs de violence conjugale ayant participé à un groupe de responsabilisation pour leurs comportements violents. Ces quatre vignettes cliniques sont tirées d'une étude longitudinale réalisée par les mêmes auteurs que la présente étude, et qui visait à évaluer ces trois caractéristiques psychologiques chez 53 sujets inscrits dans un programme de responsabilisation à Praxis. Cette étude longitudinale a été conduite de 2013 à 2017 et réunit deux associations de terrain et deux services universitaires belges et québécois : Praxis et Accord Mauricie, d'une part ; le Centre d'expertise en psychotraumatisme et psychologie légale de l'université de Liège et le Département de psychologie de l'université du Québec à Trois-Rivières, d'autre part. Les quatre participants présentés dans la présente étude clinique ont été sélectionnés en fonction de leur score à l'échelle d'alexithymie et de l'évolution de ces scores durant la prise en charge thérapeutique. 
En effet, l'objectif visé par cette étude est de vérifier l'impact de la participation à une thérapie en groupe de responsabilisation de la violence conjugale sur les changements associés à différentes caractéristiques psychologiques, dont l'alexithymie. En ce sens, ces quatre vignettes cliniques permettent de mettre en évidence différents cas de figure relatifs à l'évolution de l'alexithymie à la suite d'une prise en charge thérapeutique. La première évaluation (T1) correspond à la collecte de données avant l'entrée des participants dans le programme thérapeutique proposé par Praxis. Le second recueil (T2) est effectué au terme de celui-ci.

\section{MÉTHODE}

\section{Le programme d'accompagnement pour les auteurs de violence conjugale}

En Belgique francophone, le programme de base développé par l'association Praxis est constitué d'un minimum de $45 \mathrm{~h}$ réparties en $3 \mathrm{~h}$ de consultation individuelle et de $42 \mathrm{~h}$ de thérapie en groupe de responsabilisation : 21 séances hebdomadaires de $2 \mathrm{~h}$ (Libert, 2012). L'équipe d'intervenants est composée de psychologues, psychothérapeutes et criminologues. Les personnes bénéficiant d'une injonction de soin dans le cadre d'une procédure pénale constituent $75 \%$ de la population du service. Tandis que les autres usagers entament une démarche volontaire sur conseil d'un proche ou d'un professionnel psycho-médicosocial. Les deux clientèles sont accueillies dans les mêmes groupes. Le groupe se veut un lieu d'expérience et de réflexion sur soi. Il permet d'intervenir à plusieurs niveaux : les enjeux intrapsychiques (démarche introspective, meilleure connaissance de soi et de sa dynamique individuelle), les capacités relationnelles (à partir des interactions avec les animateurs et les pairs, mieux connaître ses stratégies dans les rapports de force, dans la négociation, dans la communication), l'environnement familial dans une approche systémique (les positionnements et les rôles endossés dans les groupes peuvent être le reflet des équivalents familiaux). Depuis ses débuts, Praxis accorde une importance particulière au développement des capacités d'expression des émotions. En 2002, Kowal détaillait les objectifs du travail socio-éducatif comme suit : «Acquérir un vocabulaire varié pour exprimer des sentiments ; apprendre à mieux percevoir ses sentiments notamment en observant ses propres réactions physiques ; déterminer les tabous, les croyances, les habitudes qui permettent ou interdisent d'exprimer certains sentiments ; mettre en évidence l'entonnoir qui existe entre la panoplie de sentiments que chacun est capable de ressentir, puis de manifester, puis de verbaliser ; développer sa capacité d'empathie : écoute de l'autre, compréhension des sentiments de l'autre, respect, etc. » (p. 63).

La responsabilisation renvoie à un processus d'appropriation des événements et de la contrainte judiciaire (Libert, 2012). La première étape de ce processus est la nécessaire reconnaissance de la réalité des actes hétéro-agressifs ainsi que leurs conséquences. La seconde étape est 1'acceptation de ses capacités décisionnelles par rapport aux actes posés et à la réparation des dommages. Ce processus est évolutif, complexe et varie en fonction du développement cognitif, de la maturité affective et relationnelle des individus (Kowal, Roussel, Deroe, Gobert, 2016).

\section{Protocole de recherche et instruments de mesure}

Outre la réalisation d'un entretien semi-structuré pour le recueil de données sociodémographiques avec chaque sujet, trois questionnaires d'auto-évaluation, correspondant à trois caractéristiques spécifiques du fonctionnement psychologique de l'individu, ont été administrés. Les cotations et interprétations ont été réalisées en respectant les règles inhérentes aux différents tests. Le score total correspond à la somme des réponses à l'ensemble des items du test administré. Tandis que le score pondéré se réfère aux sous-échelles constituant le test et correspond à la somme des réponses à chaque sous-échelle divisée par le nombre d'items qui leur est spécifique.

\section{L'échelle d'alexithymie de Toronto (Toronto} Alexithymia Scale - TAS-20)

L'échelle d'autoévaluation TAS-20 fournit une évaluation globale du niveau d'alexithymie, ainsi que trois sous-scores : difficulté à décrire les émotions (5 items), difficulté à identifier les émotions ( 7 items) et pensée opératoire ou orientée vers l'extérieur ( 8 items). Selon une conception psychopathologique, Luminet, Taylor et Bagby (2003) proposent d'utiliser les scores seuil sur l'échelle d'alexithymie, afin de mieux distinguer un individu alexithymique d'un individu dépourvu de ce trait. En effet, 1'utilisation des catégories en fonction du score total permettrait de rendre compte de la discontinuité entre les réponses normales et pathologiques. Un score plus petit ou égal à 44 signifie que le sujet est non alexithymique, un score compris entre 45 et 55 indique un fonctionnement indéterminé (sub-alexithymique), alors qu'un score plus grand ou égal à 56 signifie que le sujet est alexithymique. Les qualités métrologiques de ce questionnaire sont fiables et ont été analysées dans de nombreux travaux (Loas et coll., 2001). 
L'inventaire de dépression de Beck (Beck Depression Inventory - BDI)

Le questionnaire BDI contient 21 items et permet d'identifier des symptômes et des attitudes associés à la dépression. Le score total peut être compris entre 0 à 63 . Un score de 1 à 9 indique un niveau normal de dépression, un score de 10 à 18 indique un trouble bénin de l'humeur (cas limite de dépression), un score de 19 à 29 indique une dépression modérée et un score plus grand ou égal à 30 indique une dépression sévère.

\section{L'échelle d'impulsivité de Barratt (Barratt Impulsiveness Scale - BIS-11)}

La BIS-11 comprend trois sous-échelles : l'impulsivité motrice qui représente la dimension comportementale (11 items), l'impulsivité cognitive qui représente le processus décisionnel ( 8 items), ainsi que l'impulsivité non planifiée qui représente l'absence d'anticipation et d'orientation vers le futur (11 items). Un score plus petit ou égal à 51 indique la présence de sur-contrôle, un score de 52 à 71 correspond à un score normal et un score plus grand ou égal à 72 indique la présence d'impulsivité.

\section{RÉSULTATS}

\section{Présentation des vignettes cliniques}

Les quatre vignettes cliniques ont été sélectionnées parmi les sujets ayant participé à la fois au T1 et au T2. Les sujets présentant un score indéterminé à la TAS-20, soit en T1 soit en T2, ont été retirés de la sélection. Sur les 11 sujets dont le score $\geq 56$ à la TAS-20 en T1, 5 n'ont plus ce score en T2 et 4 l'atteignent toujours en T2. Deux vignettes ont été sélectionnées pour chaque sous-catégorie en fonction de l'intérêt des intervenants psychosociaux, d'une part, et de la précision des données cliniques à disposition, d'autre part. L'un des quatre sujets (Ali $\left.{ }^{1}\right)$ a été sélectionné d'emblée pour son caractère atypique : en effet, dans le groupe, il se montrait plutôt agité, logorrhéique et était perçu, par les animateurs, comme émotif alors qu'au terme du suivi, en prenant connaissance des cotations aux échelles de mesure, les animateurs ont découvert des résultats liés à la présence d'alexithymie et à une impulsivité normale chez ce sujet. Les trois autres sujets ont été sélectionnés afin d'observer l'éventuelle interaction entre plusieurs variables : Pierre présente une alexithymie en T1 ainsi qu'un trouble de l'humeur, Jacques présente une alexithymie en T1, mais ne présente un score significatif

1 Tous les prénoms utilisés sont fictifs, les âges et les professions ont été légèrement adaptés pour garantir l'anonymat. ni à l'échelle de dépression ni à l'échelle d'impulsivité, Rudy cumule un score élevé aux 3 variables (alexithymie, dépression et impulsivité).

L'évolution des scores à l'échelle d'alexithymie durant la prise en charge est au cœur des réflexions cliniques de cet article. En effet, deux hommes (Pierre et Jacques) obtiennent une diminution de ce score total lors du T2, c'est-à-dire au terme de leur participation au groupe de responsabilisation, tandis que les résultats des deux autres usagers (Rudy et Ali) démontrent un maintien au-delà du score seuil $\geq 56$ lors du T2 .

Pour chaque vignette, une présentation du parcours de vie de chaque individu est dressée ainsi que les résultats aux trois questionnaires d'autoévaluation et les raisons pour lesquelles ceux-ci ont été orientés vers Praxis. Enfin, les commentaires cliniques des intervenants durant le suivi sont abordés pour compléter la présentation des vignettes. Ceux-ci ont été recueillis lors des séminaires d'étude de cas organisés entre l'équipe de recherche et les intervenants psychosociaux dans un esprit d'enrichissement mutuel lors de l'analyse des résultats.

\section{Diminution du score seuil à la TAS-20 $\leq 44$ au T2 : cas de Pierre et de Jacques}

Pierre

Pierre est âgé de 52 ans. Il est mécanicien et travaille dans le secteur agricole depuis l'âge de 18 ans. Il est adressé à Praxis dans le cadre d'une mesure de médiation pénale pour des coups et blessures volontaires sur sa compagne actuelle, avec qui il entretient une relation depuis un peu plus d'un an. Il n'a pas d'antécédents judiciaires connus. Lorsque le couple se forme, il est veuf depuis à peine une année. Le décès de son épouse est survenu brutalement, après vingt ans de mariage, le laissant complètement démuni et désemparé. De cette union, il a deux fils déjà adultes. L'aîné présente des troubles du comportement qui seront ultérieurement identifiés par les intervenants comme relevant de la psychose. Pierre lui-même ne sait rien en dire. Il connaît peu la maladie de son enfant, car il s'en occupait peu jusqu'au décès de la maman. Ce dernier vit toujours au domicile familial. Les rapports entre eux sont extrêmement tendus.

Après le décès soudain de son épouse, il sombre rapidement dans l'alcoolisme. Il rencontre alors sa nouvelle compagne par les réseaux sociaux. Ellemême fait face à de nombreuses difficultés de vie (conflits familiaux avec ses enfants, conflits relationnels au travail, passé de victime de violence conjugale) et se réfugie dans l'alcool depuis de 
nombreuses années. Les épreuves et l'alcool vont souder le couple. C'est dans ce contexte que les faits de violence s'inscrivent. Pierre parle de coups et d'insultes mutuels. Lors d'un passage à l'acte, sa compagne est blessée à la tête. Elle a peur et appelle la police. Lorsque celle-ci arrive au domicile, Pierre devra être maîtrisé par la force et fera également 1'objet d'une poursuite pour rébellion sur agents. Pierre évoque une scène unique. Une récidive surviendra néanmoins pendant son accompagnement en groupe. Après l'intervention judiciaire, le couple décide d'entreprendre ensemble une démarche auprès des alcooliques anonymes. Pierre désire devenir abstinent, mais abandonnera rapidement les réunions. Cet abandon est attribué au manque de volonté de sa compagne. L'alcool reste donc un sujet de tension important dans le couple.

\begin{tabular}{lll}
\hline Variables & T1 & T2 \\
\hline $\begin{array}{l}\text { TAS-20 } \\
\text { (Alexithymie) }\end{array}$ & Score total : 59 & Score total : 42 \\
$\begin{array}{c}\text { - Difficulté } \\
\text { d'identification }\end{array}$ & $3,71(26)$ & $2,57(18)$ \\
$\begin{array}{c}\text { - Difficulté de } \\
\text { verbalisation }\end{array}$ & $3(15)$ & $1,8(9)$ \\
- Pensée opératoire & $2,25(18)$ & $1,88(15)$ \\
\hline BDI (Dépression) & Score total : 13 & Score total : 10 \\
\hline $\begin{array}{l}\text { BIS-11 (Impulsivité) } \\
\text { - Impulsivité }\end{array}$ & Score total : 55 & Score total : 51 \\
$\quad \begin{array}{l}\text { cognitive } \\
\text { - Impulsivité }\end{array}$ & $2(16)$ & $1,75(14)$ \\
$\begin{array}{l}\text { motrice } \\
\text { - Difficulté de } \\
\text { planification }\end{array}$ & $1,64(18)$ & $1,73(19)$ \\
\hline
\end{tabular}

Tableau 1. Résultats de Pierre aux trois échelles autoadministrées.

(Pour les sous-dimensions à la TAS-20 et à la BIS-11, ce sont les résultats pondérés qui sont indiqués d'abord, les résultats globaux étant indiqués entre parenthèses).

Les résultats obtenus par Pierre à la TAS-20 lors du T1 indiquent la présence d'alexithymie et, plus particulièrement, de difficultés dans l'identification et la verbalisation des émotions (voir Tableau 1). Toutefois, cette première observation se modifie au cours de la prise en charge. En effet, les résultats lors du T2 montrent une diminution du score à cette échelle indiquant l'atténuation de l'impact de cette caractéristique dans le fonctionnement psychologique du sujet avec une nette amélioration des trois sous-dimensions. Pour l'évaluation des symptômes dépressifs chez Pierre, le score total au BDI renseigne la présence d'un trouble bénin de l'humeur qui reste stable au cours du processus thérapeutique. Ce résultat signifie qu'il ne souffre pas de dépression à proprement parler, mais qu'il présente une humeur dysphorique pouvant rendre compte d'un malaise psychique. Enfin, Pierre ne présente pas d'impulsivité lors des deux évaluations réalisées. La mesure prise à la BIS-11 au T1 indique un score normal $(\leq 52)$. Toutefois, une réserve peut être émise quant à cette interprétation des résultats obtenus au T2, puisque la légère baisse du score total laisse supposer un mode de réponse sur-contrôlé (score de 51).

\section{- Observations en cours de suivi}

Dans le groupe, Pierre se montre plutôt timide et en retrait. Il s'excuse beaucoup et montre peu d'assurance dans sa prise de parole. Peu à peu, encouragé par les autres et les intervenants, il va donner accès à son histoire et ses préoccupations actuelles. La manière dont celles-ci sont livrées dénote un décryptage pauvre de son environnement confinant à la naïveté. Il semble vite perdu face à la complexité de certaines situations. Le suivi prend rapidement l'allure d'un accompagnement davantage psychoéducatif : décrypter, savoir où aller chercher des ressources.

Par rapport à ses comportements violents, Pierre exprime un fort sentiment de honte. Toutefois, il attribue leur survenance à des facteurs externes : le décès de son épouse, l'alcoolisme de sa nouvelle partenaire ou, encore, le comportement de son fils. Émotionnellement, Pierre est incapable de décrire ses affects et de faire des liens entre ce qu'il vit et ce qu'il ressent. Aussi est-ce de façon purement factuelle qu'il aborde les grands drames de sa vie. La détresse émotionnelle est pourtant bien présente. Elle se traduit chez lui par des manifestations somatiques diverses : sensation d'étouffement, vertiges. Celles-ci apparaissent dans les périodes de forte alcoolisation de sa compagne. Au cours de son accompagnement, Pierre téléphonera à deux reprises en pleurs et exprimera son envie de mettre fin à ses jours. Pourtant, une fois la crise passée, rien ne subsiste de sa détresse psychique. Rien n'est verbalisé dans l'espace groupal a posteriori. Même dans les périodes où des idéations suicidaires ont été verbalisées, Pierre ne se vit pas comme dépressif.

Au terme des 21 séances, les intervenants observent une amélioration de la capacité d'introspection et de narration. Par exemple, Pierre sera capable de faire des liens entre des événements du passé, tels que le deuil précoce de ses parents et le décès inopiné de sa femme, et le sentiment actuel de perte et d'abandon qui l'envahit lorsque sa compagne s'alcoolise.

\section{Jacques}

Jacques est âgé de 57 ans. Il est chef d'équipe dans une société de travaux publics. Dans sa 
jeunesse, il a servi plusieurs années dans l'armée où il a accompli plusieurs missions en contexte de guerre. Il vient à Praxis sous contrainte judiciaire. Il fait l'objet d'un sursis probatoire pour coups et blessures sur sa partenaire. Il a des antécédents pour des faits de violence publique. Il évoquera des bagarres lors de sorties dans les bars.

La condamnation concerne une infraction commise sur une conjointe avec qui il a entretenu une relation de trois ans. Au moment des faits, le couple était déjà séparé officiellement depuis trois mois et n'entretenait plus, selon lui, qu'une simple relation amicale. Jacques continuait à lui rendre quelques menus services. Ils s'étaient rencontrés dans un café trois ans auparavant. Jacques venait de divorcer de son épouse avec qui il était marié depuis vingt-cinq ans. De cette première union sont nés deux fils, le plus jeune souffrant d'un léger retard mental. Selon Jacques, ce nouveau couple n'a jamais bien fonctionné. La conjointe avait des rapports compliqués avec le fils cadet de Jacques en raison de son handicap. Cela créait de nombreuses tensions qui ont fini par mettre à mal la relation. Au moment de la prise en charge, il est déjà engagé avec une nouvelle compagne depuis un an et n'a plus aucun contact avec la conjointe victime de l'infraction.

\begin{tabular}{lll}
\hline Variables & T1 & T2 \\
\hline $\begin{array}{l}\text { TAS-20 } \\
\text { (Alexithymie) }\end{array}$ & Score total : 59 & Score total : 43 \\
$\begin{array}{l}\text { Difficulté } \\
\text { d'identification }\end{array}$ & $2,86(20)$ & $2(14)$ \\
$\begin{array}{l}\text { - Difficulté de } \\
\text { verbalisation }\end{array}$ & $3,2(16)$ & $2,4(12)$ \\
$\begin{array}{l}\text { - Pensée } \\
\text { opératoire }\end{array}$ & $2,86(23)$ & $2,13(17)$ \\
\hline \begin{tabular}{lll} 
BDI (Dépression) & Score total : 9 & Score total : 7 \\
\hline $\begin{array}{l}\text { BIS-11 } \\
\text { (Impulsivité) }\end{array}$ & Score total : 51 & Score total : 51 \\
- Impulsivité \\
$\begin{array}{l}\text { cognitive } \\
\text { - Impulsivité } \\
\text { motrice }\end{array}$ & $2(16)$ & $2,25(18)$ \\
- Difficulté de \\
planification
\end{tabular} & $1,73(19)$ & $1,55(17)$ \\
\hline
\end{tabular}

Tableau 2. Résultats de Jacques aux trois échelles autoadministrées.

(Pour les sous-dimensions à la TAS-20 et à la BIS-11, ce sont les résultats pondérés qui sont indiqués d'abord, les résultats globaux étant indiqués entre parenthèses).

Quant aux faits, Jacques évoque une scène de violence unique dont il n'a aucun souvenir. Il se défend toutefois d'avoir été sous l'effet de l'al- cool et il dit n'avoir consommé que cinq bières ce jour-là. Le rapport de police auquel il a eu accès rapporte des bousculades et des coups au visage. L'état de la conjointe a nécessité une prise en charge médicale. Durant cette altercation violente, le fils cadet de Jacques était également présent. Jacques a tendance à s'appuyer sur l'absence de souvenir pour maintenir l'ambivalence quant à l'intensité de ses comportements violents et leurs impacts. Il ne se reconnaît aucun problème avec l'alcool. L'ampleur réelle de sa consommation semble minimisée.

Le TAS-20 indique un trouble alexithymique en $\mathrm{T} 1$ avec une difficulté à identifier ses émotions qui serait associée à une pensée opératoire (voir Tableau 2). Le résultat du T2 montre une absence d'alexithymie avec une amélioration sur les trois sous-dimensions. Le BDI montre un score normal en $\mathrm{T} 1$ et $\mathrm{T} 2$, ce qui signifie que le sujet ne souffre d'aucun trouble de l'humeur. Le T1 et le T2 à l'échelle de Barratt indiquent un score $\leq 51$, ce qui peut laisser supposer un mode de réponse sur-contrôlé.

\section{- Observations en cours de suivi}

Dans le groupe, Jacques apparaît d'emblée affable et jovial. On le sent à l'aise dans les interactions sociales. Il semble disposer d'une certaine habileté à gérer les relations sociales simples avec bon sens et bienveillance, habilité qu'il dit avoir acquise dans l'exercice de sa profession. Les conseils dispensés aux autres traduisent, toutefois, une compréhension concrète et normative du monde. Il semble en effet éprouver certaines difficultés lorsqu'il s'agit de décoder la dimension émotionnelle de certaines situations et des relations interpersonnelles en général.

Pour lui-même, il n'exprime aucune souffrance et ne porte aucune demande particulière. Il est engagé dans une nouvelle relation amoureuse qu'il décrit comme sereine et satisfaisante et se dit épanoui sur le plan familial et professionnel. Il adhérera néanmoins sans difficulté à la proposition des intervenants de questionner ses besoins et attentes sur le plan affectif.

Concernant ses comportements violents, il se réfugiera tout au long du suivi derrière l'absence de souvenirs, qui semble ne lui procurer aucun inconfort, mais avoir pour fonction de maintenir une dénégation des actes posés. Il lui sera donc impossible de décoder les éléments déclencheurs de la violence conjugale.

Si le groupe a été vécu par Jacques comme une expérience sociale positive, l'accompagnement n'a pas permis l'émergence d'une demande d'aide ou de changement propre. 
Maintien d'un score seuil $\geq 56$ à la TAS-20, en T2 : le cas de Rudy et de Ali

\section{Rudy}

Rudy est âgé de 46 ans. Il travaille comme ouvrier dans une société de travaux publics, mais est en incapacité de travail depuis deux ans, en suite de plusieurs accidents vasculaires cérébraux dont il garde des séquelles : motricité ralentie, vision et audition diminuées.

Rudy est référé dans le cadre d'une peine correctionnelle avec sursis probatoire. L'obligation de suivi fait partie des conditions. Il y a quelques années, une première tentative de prise en charge à Praxis, intervenant également dans un cadre judiciaire, n'avait pas abouti, suite à un abandon. Rudy a déjà effectué plusieurs séjours en détention préventive. Tous les faits concernent la même compagne. En effet, les violences s'inscrivent dans une relation conjugale de plus de dix ans. Il s'agit d'une relation chaotique émaillée de nombreuses séparations. Le couple n'a pas d'enfant commun. Rudy a un fils de 20 ans, d'une précédente union, qu'il ne voit plus depuis neuf ans. Madame a deux filles vivant au domicile conjugal.

La violence est présente dans le couple depuis le début de la relation. Rudy reconnaît des passages à l'acte fréquents : violence verbale (insultes), psychologique (dénigrement), physique (coups, strangulation) et bris d'objets. Les comportements violents se déroulent majoritairement dans un contexte de forte alcoolisation. Rudy consomme depuis l'âge de 16 ans. Il y a eu plusieurs tentatives de cure qui n'ont pas abouti.

\begin{tabular}{lll}
\hline Variables & T1 & T2 \\
\hline $\begin{array}{l}\text { TAS-20 } \\
\text { (Alexithymie) }\end{array}$ & Score total : 58 & Score total : 80 \\
$\begin{array}{c}\text { - Difficulté } \\
\text { d'identification }\end{array}$ & $3,29(23)$ & $4,14(29)$ \\
$\begin{array}{c}\text { - Difficulté de } \\
\text { verbalisation }\end{array}$ & $2,6(13)$ & $4,6(23)$ \\
$\begin{array}{c}\text { - Pensée } \\
\text { opératoire }\end{array}$ & $2,75(22)$ & $3,5(28)$ \\
\hline BDI (Dépression) & Score total : 23 & Score total : 24 \\
\hline $\begin{array}{c}\text { BIS-11 } \\
\text { (Impulsivité) }\end{array}$ & Score total : 71 & Score total : 83 \\
- Impulsivité \\
$\begin{array}{c}\text { cognitive } \\
\text { - Impulsivité } \\
\text { motrice }\end{array}$ & $2,63(21)$ & $3,25(26)$ \\
$-\begin{array}{c}\text { Difficulté de } \\
\text { planification }\end{array}$ & $2,18(24)$ & $2,73(30)$ \\
\hline
\end{tabular}

Tableau 3. Résultats de Rudy aux trois échelles autoadministrées.

(Pour les sous-dimensions à la TAS-20 et à la BIS-11, ce sont les résultats pondérés qui sont indiqués d'abord, les résultats globaux étant indiqués entre parenthèses).
Les résultats à la TAS-20 sont élevés et révèlent une alexithymie qui s'accentue en T2 (voir Tableau 3). Les difficultés à identifier ses émotions et à les exprimer se maintiennent malgré la prise en charge. Le BDI révèle une dépression modérée qui persiste en T2. La BIS-11 mesure un score normal en T1, mais la présence d'impulsivité en T2 avec une accentuation de l'impulsivité cognitive et de la difficulté à planifier (anticiper et s'orienter vers le futur). Ainsi, il y a une accumulation de trois problématiques : alexithymie, dépression et impulsivité.

La répétition des agissements violents, malgré les condamnations judiciaires ainsi que la répétition des rechutes alcooliques, viennent compléter le tableau clinique et conduisent à poser l'hypothèse d'une grande souffrance psychique.

— Observations en cours de suivi

Dans le groupe, Rudy apparaît réservé, voire timoré. Il prend rarement la parole de lui-même. Il reste très discret sur son parcours de vie. On obtiendra peu d'informations sur son histoire familiale. Seules les difficultés de couple en lien avec la gestion financière, le partage des tâches et la cohabitation inhérente aux familles recomposées sont évoquées spontanément, mais de façon très factuelle. Rudy se montre incapable d'aborder la dimension affective de sa relation de couple. L'impact sur les enfants est occulté. Ces derniers sont par ailleurs absents de son discours.

Les seules thématiques abordées à minima sont relatives à sa santé. Rudy est convaincu d'avoir une espérance de vie réduite, en raison d'un environnement professionnel extrêmement pénible et toxique et un alcoolisme dont il souffre depuis l'adolescence. Il en vient à décrire cette problématique comme une fatalité : « ça fait partie de ma vie ».

Pendant les séances, Rudy adopte une posture amorphe. Il ne verbalise aucun affect, aucune anxiété. Il peut même apparaître hébété et sa perception du monde reste factuelle. Il a peu accès à son monde intérieur. En séance, il recherche rarement les interactions avec les autres membres du groupe.

Depuis l'échec de sa dernière prise en charge, Rudy a entamé une nouvelle prise en charge médicale pour son alcoolisme, même si l'abstinence n'est pas maintenue. Il est en incapacité de travail et donc davantage disponible. Le suivi, au détour d'une activité de groupe, lui permettra d'exprimer une certaine nostalgie par rapport à une jeunesse insouciante idéalisée.

Au cours de son accompagnement, Rudy aura suscité chez les intervenants l'ennui et un grand 
sentiment d'impuissance. Au terme des 21 séances, l'inquiétude demeure quant aux probabilités de rechute alcoolique ou de récidive violente. Les facteurs de protection restent difficiles à identifier. Même si l'accompagnement a permis d'ouvrir à quelques expressions socio-affectives comme l'humour ou la nostalgie, il n'aura pas été suffisant pour susciter une demande d'aide plus consistante, malgré les propositions du groupe et des intervenants de maintenir sa participation dans le groupe ou de bénéficier d'un suivi individuel.

\section{Ali}

Ali est âgé de 31 ans. Il est d'origine maghrébine. Primo arrivant, il vit et travaille sur le territoire belge depuis cinq ans. Il est employé à temps plein dans une société de nettoyage, activité professionnelle en deçà de ses compétences réelles, puisqu'il se dit diplômé de l'enseignement supérieur dans son propre pays. Il a un parcours migratoire solitaire et se trouve donc, en Belgique, fort isolé familialement. Sa situation est également extrêmement précaire puisque son titre de séjour est arrivé à expiration et il vient de recevoir une ordonnance d'expulsion du territoire, contre laquelle il a introduit un recours. C'est par ce contexte de stress aigu qu'Ali justifie sa consommation excessive de caféine et de nicotine.

Ali fait l'objet d'une mesure de médiation pénale pour des coups et blessures volontaires à l'égard de son ex-compagne. Ils sont séparés depuis plus de six mois et une procédure de divorce est en cours. Celle-ci fut initiée par la conjointe à la suite de la répétition des comportements violents au sein du couple. Pour lui, elle est la conséquence de multiples tromperies de sa compagne, du rejet et de la malveillance de la belle-famille à son égard.

La relation a duré trois ans. L'ex-compagne d'Ali est de culture arabo-musulmane, mais est née en Belgique. Le couple n'a pas d'enfants. Ali évoque une fausse couche quelques mois après le mariage. Lorsque le suivi débute, et contre toute vérité, Ali est convaincu qu'au moment de la séparation, sa conjointe était enceinte. Il est persuadé que cette grossesse a été menée à son terme et qu'on le prive à dessein de l'exercice de sa paternité.

Ali fait l'objet de plusieurs plaintes y compris pour harcèlement et menace à la suite de la séparation. Il reconnaît à minima la violence verbale, quelques gifles appuyées et des objets cassés. Cette violence est, selon lui, la résultante de l'hostilité, la malhonnêteté et l'agressivité des autres à son égard. Il se vit avant tout comme une victime et continue à nourrir des scénarios de vengeance, tant à l'en- contre de son ex-conjointe, de sa famille que de son supposé amant.

\begin{tabular}{|c|c|c|}
\hline Variables & $\mathrm{T} 1$ & $\mathrm{~T} 2$ \\
\hline TAS-20 (Alexithymie) & Score total : 68 & Score total : 61 \\
\hline $\begin{array}{l}\text { - Difficulté } \\
\text { d'identification }\end{array}$ & $4,14(29)$ & $3(21)$ \\
\hline $\begin{array}{l}\text { - Difficulté de } \\
\text { verbalisation }\end{array}$ & $3,2(16)$ & $3,4(17)$ \\
\hline - Pensée opératoire & $2,88(23)$ & $2,88(23)$ \\
\hline BDI (Dépression) & Score total : 20 & Score total : 19 \\
\hline BIS-11 (Impulsivité) & Score total : 71 & Score total : 69 \\
\hline $\begin{array}{l}\text { - Impulsivité } \\
\text { cognitive }\end{array}$ & $2,88(23)$ & $2,25(18)$ \\
\hline - Impulsivité motrice & $2,45(27)$ & $2,27(25)$ \\
\hline $\begin{array}{l}\text { - Difficulté de } \\
\text { planification }\end{array}$ & $1,19(21)$ & $2,36(26)$ \\
\hline
\end{tabular}

Tableau 4. Résultats d'Ali aux trois échelles autoadministrées.

(Pour les sous-dimensions à la TAS-20 et à la BIS-11, ce sont les résultats pondérés qui sont indiqués d'abord, les résultats globaux étant indiqués entre parenthèses).

Le sujet présente une alexithymie en T1 comme en T2 (voir Tableau 4). On constate néanmoins une légère amélioration en $\mathrm{T} 2$ dans la capacité à identifier les émotions. Le résultat au BDI indique une dépression modérée en T1 comme en T2. La BIS-11 mesure un score normal d'impulsivité. Le sujet cumule donc deux problématiques : l'alexithymie et la dépression. Au terme de l'accompagnement, on ne constate aucune amélioration notable sur ces deux dimensions.

\section{- Observation en cours de suivi}

Dans le groupe, Ali prend d'emblée une place importante. Il a besoin de s'exprimer et monopolise rapidement l'espace groupal. Il est difficile à contenir. Il semble en grande souffrance psychique et manifeste des signes d'anxiété évidents. Il présente quelques affects dépressifs peu verbalisés, mais qui se lisent physiquement. La palette des émotions exprimées est restreinte à la colère, voire à la rage.

Dans un premier temps, Ali suscite l'écoute attentive et compréhensive de la part des autres membres du groupe, son histoire intrigue. Mais rapidement, son discours va prendre une tournure obsessionnelle allant parfois jusqu'à l'absurde. Son discours est de plus en plus décousu et logorrhéique.

L'empathie qu'il suscitait au sein du groupe va alors doucement se muer en agacement, méfiance, voire rejet. Le groupe semble, malgré tout, un point d'ancrage dans une certaine réalité. Il peut y déposer ses angoisses. Les intervenants décident donc de poursuivre le suivi avec les objectifs de renforcer cet ancrage, de l'aider à distinguer les émotions liées à 
des événements présents, des émotions liées à des événements anticipés ou fantasmés. Le travail d'accompagnement va se focaliser sur les pertes réelles et objectivables (1'expiration de son titre de séjour, le risque de perdre son emploi qui en découle, la peur d'un retour forcé à son pays d'origine), tout en le soutenant dans des stratégies adéquates de récupération de ses droits.

Peu à peu, l'aspect quasi délirant du discours va s'estomper. Ali va s'apaiser et donner accès à ses enjeux narcissiques : la peur de l'échec, le sentiment d'humiliation, l'impossible retour au pays d'origine et la perte de statut social qui en découlerait pour lui et sa famille. En perdant son lien marital, il perd son droit à une vie meilleure en Europe. Elle le prive aussi d'une possible paternité, gage de sécurité et pérennité pour lui sur le territoire belge. Il va également, progressivement, donner accès à son histoire familiale, son parcours migratoire solitaire et risqué, son parcours d'intégration complexe. La question de la violence agie au sein du couple reste minimisée.

\section{DISCUSSION}

Ces quatre vignettes cliniques présentent des points communs, mais également des différences marquantes. Tous les sujets présentent au T1 un trouble alexithymique. Toutefois, deux d'entre eux, Pierre et Jacques, n'atteignent plus le score seuil $\geq 56$ à l'échelle du TAS-20 lors du T2, mais atteignent un score en dessous du seuil $\leq 44$, alors que pour les deux autres, Rudy et Ali, le score seuil $\geq 56$ reste atteint. Pierre est le seul sujet à prolonger l'accompagnement au-delà des heures imposées dans le cadre judiciaire.

Pour Pierre, Jacques et Rudy, les observations cliniques corroborent l'expression d'un trouble alexithymique. Cela signifie qu'en début de suivi, les sujets présentent, de manière générale, des difficultés à identifier leurs propres sentiments, à les décrire aux autres, et ont tendance à recourir à une forme de pensée de type opératoire. Ils produisent des récits plutôt stéréotypés avec une pauvreté de mots. Ils montrent une tendance à décrire les circonstances entourant les événements en les vidant de leurs affects.

L'expression de l'alexithymie d'Ali est plus inattendue. En effet, même si la stéréotypie s'observe dans une rigidité des pensées automatiques et des analyses formatées par des normes culturelles, le groupe est plutôt confronté aux récits débridés du sujet, dans une élaboration échappant parfois à la rationalité. Il semble d'ailleurs difficile de formuler des hypothèses quant à la vie fantasmatique du sujet. Celui-ci semble manifester une vie fantasmatique importante, mais orientée vers un environnement extérieur vécu comme menaçant ou persécutant. Le décodage des événements est perturbé par des distorsions cognitives importantes, ainsi qu'un déficit de relation logique et temporelle entre sensations physiques, état émotionnel et pensées. Dans ce cas clinique précis, il aurait été utile de recueillir des données complémentaires quant aux traits de personnalité. Dans la réalité concrète de l'accompagnement, considérer la logorrhée, l'instabilité émotionnelle et l'agitation motrice d'Ali, comme les signes d'un épisode de décompensation, a permis aux intervenants de persévérer dans la prise en charge et de maintenir un lien empathique minimal entre le groupe et le sujet. Avoir poursuivi le programme jusqu'au terme de l'obligation judiciaire permet à l'usager de compléter, de manière réelle et concrète, une action positive qui répare une partie de ses pertes. Le sujet lui-même reconnaît avoir appris à « mieux se gérer ». Ceci peut expliquer, en partie, la légère amélioration de la sous-dimension concernant l'identification des émotions, même si les résultats à la TAS-20 et au BDI ne montrent aucune amélioration globale significative des troubles alexithymique et dépressif.

Un autre point commun entre les quatre vignettes est la consommation d'alcool ou de substances excitantes. Concernant les troubles de l'usage de l'alcool, Praxis se réfère aux catégories définies par la Société française d'alcoologie (SFA) et la Société scientifique de médecine générale (SSMG) en Belgique francophone. Les troubles liés à l'usage de l'alcool, représentés par l'usage nocif et l'usage avec dépendance, correspondent aux formes symptomatiques de l'usage, c'est-à-dire qui se traduisent par des conséquences visibles sur le plan social, psychologique ou médical. Il n'y a pas de dépistage systématique pour les participants des groupes à Praxis, mais une attention particulière accordée à ce facteur de risque abordé avec tous les participants, dans tous les groupes. Les observations cliniques permettent de différencier la fonction de la substance psychotrope chez les quatre sujets présentés ici. Pierre et Jacques présentent une surconsommation circonstancielle ou récente. Il reste une énigme quant à l'ampleur réelle de la consommation d'alcool de Jacques et de sa fonction notamment au vu du phénomène quasi dissociatif décrit au moment des comportements violents. Pierre se montre davantage dans un mouvement de prise de conscience de la consommation problématique au sein de son couple et est à la recherche de solutions. Chez Rudy, la détresse psychique se manifeste par une symptomatologie variée, les plaintes somati- 
ques sont nombreuses. La consommation d'alcool vient suppléer les affects dépressifs et ce, depuis de nombreuses années. L'impulsivité mesurée en T2 chez Rudy pourrait confirmer les inquiétudes des intervenants quant à une possible rechute alcoolique ou récidive à la fin du suivi. Chez Ali, la détresse psychique se manifeste par une agitation verbale et motrice importante, qui est stimulée par une surconsommation de produits excitants (une consommation extrêmement importante de tabac et de café). Il est étonnant qu'aucun indice d'impulsivité motrice ou cognitive ne vienne corroborer ces observations.

À partir de ces vignettes se dessinent deux portraits cliniques distincts, selon l'étiologie différenciée rappelée notamment par Loas (2010) et Jouanne (2006), et présentée précédemment dans les éléments de définition. Selon l'approche qui se réfère au modèle de fonctionnement défensif, l'hypothèse d'une alexithymie secondaire peut être retenue pour Pierre et Jacques. En revanche, pour Rudy et Ali, il pourrait s'agir d'une alexithymie primaire et stable, intégrée dans la structure de personnalité, comme le suggère le modèle déficitaire.

En effet, Pierre et Jacques ont comme point commun d'avoir commis des coups et blessures volontaires sur leur conjointe dans un contexte de deuil récent (pour Pierre) ou de perte significative (un divorce après 25 ans de mariage, pour Jacques). Le contexte des passages à l'acte se caractérise également, dans les deux cas, par un stress important vécu depuis plusieurs mois, en lien avec un nouveau rôle parental auprès d'un enfant nécessitant des soins spécifiques. Selon Jouanne (2006), le verrouillage psychologique de la régulation des émotions constitue une stratégie adaptative face à une situation de stress aigu. « La conduite addictive constituerait un aménagement défensif compensateur du fait de la faiblesse des mécanismes de défense et de l'incapacité du moi à réguler et moduler les émotions » (Jouanne, 2006, p. 197). La prise en charge thérapeutique a pu avoir pour effet d'aider les sujets à renoncer, au moins partiellement, à ces stratégies, qui les maintenaient dans un équilibre de plus en plus précaire, et à élaborer des alternatives moins défensives. Les résultats en T2 à la TAS-20 montrent chez ces deux sujets une amélioration du score global, mais également des trois sous-dimensions. La prise en charge thérapeutique a pu susciter le développement de la capacité d'empathie et des capacités de mentalisation, se traduisant par une amélioration sur le plan de l'alexithymie.

Pierre présente un trouble bénin de l'humeur. Le déverrouillage émotionnel observé et mesuré a, sans doute, eu pour effet de le connecter davantage à une souffrance psychique. Il formulera davantage de plaintes somatiques, manifestera de la détresse et le besoin de soutien. Par contre, Jacques ne présente ni en T1, ni en T2 un trouble de l'humeur ou une impulsivité problématique. L'accompagnement a donc permis une sorte de rétablissement des capacités de régulation émotionnelle, sans susciter un prolongement de la demande d'aide. Ses antécédents judiciaires laissent supposer que ce n'est pas la première fois que Jacques traverse ce type de déséquilibre. L'impact de son passé militaire sur ses modes de stratégie relationnelle en situation de stress émotionnel n'a pas été évalué.

Pour ce qui est de Rudy et Ali, leur parcours dévoile des passages à l'acte violents fréquents et de nature variée. Dans les deux cas, nous observons la coexistence du trouble alexithymique et d'un trouble dépressif. Leur capacité d'empathie et d'élaboration psychique est et reste réduite. Pour toutes ces raisons, les observations cliniques conduisent à formuler l'hypothèse d'une alexithymie primaire conçue comme un déficit structuré et durable du fonctionnement préconscient. Même si Zimmermann, Salamin et Reicherts (2008) invitent à une lecture rigoureuse et prudente des études en la matière, l'équipe de recherche produit des résultats significatifs quant à la relation stable entre l'alexithymie et plusieurs facettes de la personnalité. Selon ces auteurs, l'alexithymie, en tant que trait stable, augmente la vulnérabilité au stress et aux situations chargées émotionnellement, tout en amenant l'individu à s'exposer plus fréquemment à des situations à risque. Chez ces individus, l'agir (abus de substances, transgression, etc.) leur permet de diminuer leurs tensions corporelles. Le maintien en T2 des troubles alexithymiques et dépressifs suggèrent donc une difficulté à introduire un changement thérapeutique, d'autant plus sans obligation de soin.

\section{CONCLUSION}

La pratique clinique confirme la difficulté de prise en charge des sujets alexithymiques et auteurs de violence conjugale. En effet, les intervenants psychosociaux formulent rapidement un sentiment d'impuissance dans l'accompagnement de ces personnes qui expriment peu de demandes d'aide.

Par exemple, il est frustrant pour les intervenants de constater que Rudy et Ali ne formulent pas de demande d'aide au-delà de la prise en charge obligatoire, alors que les facteurs de risque tels que l'isolement de l'auteur ou du couple, le manque d'accès à des soins appropriés, un sentiment d'échec ou un 
manque d'espoir, l'absence d'empathie, des ruminations de vengeance vis-à-vis de la partenaire sont encore présents et semblent générer une souffrance psychique (Drouin et coll. 2012).

Cependant, la différenciation entre alexithymie primaire et secondaire semble une distinction pertinente pour le travail clinique, car elle permet aux intervenants d'ajuster leurs attentes. Il est encourageant de constater que les personnes présentant une alexithymie de type secondaire peuvent améliorer, lors de la prise en charge, leur capacité à identifier leurs émotions, à les verbaliser et à les intégrer dans des stratégies relationnelles plus adéquates.

Quelle que soit la nature de l'alexithymie, la méthode de groupe a permis, dans ces quatre cas précis, d'aider à la fois l'usager et les intervenants à persévérer dans l'accompagnement, au moins jusqu'au terme du suivi obligatoire. Le groupe a parfois pallié le déficit d'expression émotionnelle et de mentalisation, tout en acceptant la personne avec bienveillance et en contenant les phases d'émois éventuellement anxiogènes.

En revanche, le cumul des problématiques, la présence d'antécédents judiciaires ou d'une symptomatologie complexe et ancienne pourraient constituer des indicateurs pertinents pour orienter les intervenants vers une hypothèse d'alexithymie primaire. Une anamnèse plus fouillée des antécédents, ainsi qu'un diagnostic de personnalité sont deux perspectives cliniques à explorer afin d'améliorer la prise en charge des personnes souffrant d'un trouble alexithymique stable. D'un autre côté, une hypothèse peut être émise quant au maintien de 1'alexithymie au T2. En effet, la décharge pulsionnelle dans l'agir pourrait permettre à l'individu de préserver un état d'équilibre émotionnel. L'individu ne pouvant exprimer ou élaborer verbalement ses pulsions aurait tendance à utiliser la voie comportementale, notamment par des agissements violents. Ainsi, un travail axé sur l'élaboration verbale pourrait nécessiter la mise en place d'un suivi individuel à plus long terme, pour pouvoir observer une diminution de l'alexithymie primaire chez l'individu. Dans cette perspective, il serait pertinent d'explorer comment les méthodes thérapeutiques individuelles et de groupes peuvent se compléter ou se renforcer.

Dans une étude menée auprès de trente-quatre hommes auteurs de violence conjugale pris en charge dans un service spécialisé, Léveillée et coll. (2009) explorent les comportements autodestructeurs au sein de cette population. Les résultats indiquent que 73,5\% de l'échantillon manifestent ou ont manifesté de l'autodestruction au cours de leur vie (idées suicidaires, tentatives de suicide, automutilation). De plus, les résultats révèlent que les comportements autodestructeurs de ces hommes sont davantage teintés d'hostilité et non d'une évaluation négative de soi. Une première perspective de recherche serait d'évaluer les corrélations existant entre la présence d'un trouble alexithymique et dépressif avec ou sans conduites autodestructrices. L'importance d'étudier les enjeux autodestructeurs de cette clientèle est soulignée autant par les cliniciens que par les chercheurs. De plus, se pose la question du prolongement des obligations de soin et de l'intervention pour les hommes cumulant plusieurs facteurs de risque, afin de les aider à formuler une demande d'aide spontanée et pour mieux explorer la détresse psychique sous-jacente aux comportements violents au sein du couple.

Enfin, une autre perspective de recherche consisterait à comparer la régulation émotionnelle chez des femmes victimes de violence conjugale et des hommes auteurs de violence conjugale. En effet, une étude de Cavanaugh et coll. (2012) indique la présence d'alexithymie et d'affects dépressifs chez les femmes victimes de violence conjugale. Selon Luminet, Vermeulen et Grynberg (2013), les hommes ne sont pas nécessairement plus alexithymiques que les femmes. Toutefois, l'examen des sous-dimensions indique une plus grande prévalence chez les hommes des dimensions «difficulté à décrire les sentiments » et «pensée opératoire ». 


\section{RÉFÉRENCES}

Adshead (Gwen), Moore (Estelle), Humphrey (Martin), WILSON (Claire), TAPP (James).- The role of mentalising in the management of violence, Advances in Psychiatric Treatment, 19, 1, 2013, p. 67-76.

Bateman (Anthony), Fonagy (Peter).- Mentalisation et trouble de la personnalité limite. Guide pratique, Louvain-la-Neuve, De Boeck Supérieur, 2015.

Bréjard (Vincent), Bonnet (Agnès), Pedinielli (JeanLouis).- Régulation des émotions, dépression et conduites à risques : 1'alexithymie, un facteur modérateur, Annales médico-psychologiques, 166, 4, 2008, p. 260-268.

Cavanaugh (Courtenay E.), Messing (Jill T.), Petras (Hanno), Fowler (Barbara), LA Flair (Lareina), Kub (Joan), Agnew (Jacqueline), Fitzgerald (Sheila), Bolyard (Richelle), Campbell (Jacquelyn C.).-- Patterns of Violence Against Women: A Latent Class Analysis, Psychological Trauma: Theory, Research, Practice, and Policy, 4, 2, 2012, p. 169-176.

Corcos (Maurice), Pirlot (Gérard), LoAs (Gwenolé).Qu'est-ce que l'alexithymie ?, Paris, Dunod, 2011.

Corcos (Maurice), Speraza (Mario), Jeammet (Philippe).- Psychopathologie de l'alexithymie, Paris, Dunod, 2003.

Di Piazza (Laetitia), Kowal (Cécile), Hodiaumont (Fabienne), LéveILlée (Suzanne), Touchette (Lysianne), Ayotte (Robert), Blavier (Adélaïde).- Étude sur les caractéristiques psychologiques des hommes auteurs de violences conjugales : quel type de fragilité psychique le passage à l'acte violent dissimule-t-il ?, Annales médicopsychologiques, 175, 8, 2017, p. 698-704.

Drouin (Christine), Lindsay (Jocelyn), Dubé (Myriam), TréPanier (Mario), Blanchette (Daniel).- Intervenir auprès des hommes pour prévenir l'homicide conjugal (Outils no 10). Montréal, QC, Québec, QC, CRI-VIFF. 2012. [Consulté le 15 mai 2019 à http://www.criviff. qc.ca/cms/liste_publications2.php?id=21\&lang=fr]

FreYberger (Hellmuth).- Supportive psychotherapeutic techniques in primary and secondary alexithymia/ discussion, Psychotherapy and Psychosomatics, 28, 1-4, 1977, p. 337-345.

Frye-Cox (Nick E.), Hesse (Colin R.).- Alexithymia and marital quality: The mediating roles of loneliness and intimate communication, Journal of Family Psychology, 27, 2, 2013, p. 203-211.

Guilbaud (Olivier), Loas (Gwenolé), Corcos (Maurice), Speranza (Mario), Stephan (Philippe), PerezDiaz (Fernando), Venisse (Jean-Luc), Guelfi (Julien D.), Bizouard (Paul), Lang (François), Flament (Martine), Jeammet (Philippe).- L'alexithymie dans les conduites de dépendance et chez le sujet sain : valeur en population française et francophone, Annales médico-psychologiques, 160, 1, 2002, p. 77-85.

Honkalampi (Kirsi), Hintikka (Jukka), Tanskanen
(Antti), Lehtonen (Johannes), VinAmÄKI (Heimo).Depression is strongly associated with alexithymia in the general population, Journal of Psychosomatic Research, 48, 1, 2000, p. 99-104.

JouAnne (Céline).- L'alexithymie : entre déficit émotionnel et processus adaptatif, Psychotropes, 3, 12, 2006, p. 193-209.

Kowal (Cécile).- Le travail en groupe avec des auteurs de violences conjugales sous mandat judiciaire, L'observatoire : revue d'action sociale et médico-sociale, 34, 2002, p. 62-68.

Kowal (Cécile), Roussel (Mathieu), Deroe (Erwin), Gobert (Valérie).- L'évaluation des auteurs de violences conjugales, dans Coutanceau (R.), Salmona (M.), Violences conjugales et familles, Paris, Dunod, 2016, p. 183-196.

Léveillée (Suzanne), Lefebvre (Julie), Ayotte (Robert), Marleau (Jacques D.), Forest (Martin), Brisson (Martin).- L'autodestruction chez des hommes qui commettent de la violence conjugale, Bulletin de psychologie, 62, 6, 2009, p. 543-551.

Léveillée (Suzanne), Touchette (Lysianne), Ayotte (Robert), Blanchette (Daniel), Brisson (Martin), Brunelle (Alain), Turcotte (Claude).- Le changement psychologique des hommes qui exercent de la violence conjugale, Revue québécoise de psychologie, 34, 1, 2013, p. 73-94.

LIBERT (Vincent).- Praxis et le travail de responsabilisation auprès des auteurs de violences conjugales et intrafamiliales, dans Libert (V.), Jacob (A.), Kowal (C.), L'aide aux auteur(e)s de violences conjugales et intrafamiliales, Louvain-La-Neuve, éditions Academia, L'Harmattan, 2012, p. 15-51.

LoAs (Gwenolé).- L'alexithymie, Annales médico-psychologiques, 168, 9, 2010, p. 712-715.

Loas (Gwenolé), Corcos (Maurice), Stephan (Philippe), Pellet (Jacques), Bizouard (Paul), Venisse (Jean-Luc), Perez-Diaz (Fernando), Guelfi (Julien D.), JEAMmET (Philippe).- Factorial structure of the 20-item Toronto Alexithymia Scale: Confirmatory factorial analyses in non-clinical and clinical samples, Journal of Psychosomatic Research, 50, 5, 2001, p. 255-61.

Luminet (Olivier), TAYLOR (Graeme J.), BAGBY (Michael R.).- La mesure de l'alexithymie, Psychopathologie de l'alexithymie, 2003, p. 183-204.

Luminet (Olivier), Vermeulen (Nicolas), Grynberg (Delphine).-L'alexithymie, Bruxelles, De Boeck, 2013.

Maisondieu (Jean), Tarrieu (C.), Razafimamonjy (J.), Arnault (M).-- Alexithymie, dépression et incarcération prolongée, Annales médico-psychologiques, 166, 8, 2008, p. 664-668.

Ministère de la SÉCurité PUblique.- Statistiques 2013 sur la criminalité commise dans un contexte conjugal au Québec, 2013, [Consulté le 15 mai 2019 à http://www. 
securitepublique.gouv.qc.ca/police/publications-et-statistiques/violence-conjugale/2013/infractions.html]

Ogrodniczuk (John S.), Sochting (Ingrid), PiPer (William E.), Joyce (Anthony S.).- A naturalistic study of alexithymia among psychiatric outpatients treated in an integrated group therapy program, Psychology and Psychotherapy, 85, 3, 2012, p. 278-291.

Ordre des psychologues au Québec [Consulté le 15 mai 2019 à https://www.ordrepsy.qc.ca]
Tychey (Claude de).- Alexithymie et pensée opératoire : approche comparative clinique projective francoaméricaine, Psychologie clinique et projective, 1, 16, 2010, p. 177-207.

Zimmermann (Grégoire), Salamin (Virginie), ReichERTS (Michael).- L'alexithymie aujourd'hui : essai d'articulation avec les conceptions contemporaines des émotions et de la personnalité, Psychologie française, 53, 1, 2008, p. 115-128. 